\title{
A NEW VIEW ON ONE PROBLEM OF ASYMPTOTIC BEHAVIOR OF SOLUTIONS OF DELAY DIFFERENCE EQUATIONS
}

\author{
L. SHAIKHET
}

Received 20 January 2006; Accepted 24 March 2006

One known theorem on the asymptotic behavior of solution of linear delay difference equation is considered where a stability criterion is derived via a positive root of the corresponding characteristic equation. Two new directions for further investigation are proposed. The first direction is connected with a weakening of the known stability criterion; the second one is connected with consideration of negative and complex roots of the characteristic equation. A lot of pictures with stability regions and trajectories of considered processes are presented for visual demonstration of the proposed directions.

Copyright (C) 2006 L. Shaikhet. This is an open access article distributed under the Creative Commons Attribution License, which permits unrestricted use, distribution, and reproduction in any medium, provided the original work is properly cited.

\section{Introduction: statement of the problem}

There is a series of papers (see, e.g., [5-12]) where a similar method is used for investigation of asymptotic behavior of solutions of difference equations [5, 7], and differential equations $[9,10,12]$, integro-differential equations $[6,8]$, and difference equations with continuous time [11]. The basic assumption in this method is that the positive root of the corresponding characteristic equation satisfies a special sufficient condition for asymptotic stability of some auxiliary equation. Here on the example of Volterra difference equation it is proposed to improve the results of these investigations in two directions. Firstly it is shown that the basic assumption on the positive root of the corresponding characteristic equation can be essentially weaken using different conditions for asymptotic stability. Besides of that it is shown that consideration of negative and complex roots of the characteristic equation gives some new horizons for investigation. For visual demonstration of the proposed ideas, a lot of pictures with numerical calculations of stability regions and trajectories of considered processes are presented.

Consider the Volterra difference equation

$$
\Delta x_{n}=a x_{n}+\sum_{j=1}^{\infty} K_{j} x_{n-j}, \quad n \geq 0
$$

Hindawi Publishing Corporation

Discrete Dynamics in Nature and Society

Volume 2006, Article ID 74043, Pages 1-16

DOI 10.1155/DDNS/2006/74043 
2 A new view on one problem

with the initial condition

$$
x_{j}=\phi_{j}, \quad j \leq 0 .
$$

Here $\Delta x_{n}=x_{n+1}-x_{n}, a$ and $K_{j}, j=1,2, \ldots$, are real numbers. The equation

$$
\lambda-1=a+\sum_{j=1}^{\infty} \lambda^{-j} K_{j}
$$

is called the characteristic equation of difference equation (1.1).

THeOREM 1.1. Let $\lambda_{0}$ be a positive root of characteristic equation (1.3) with the property

$$
\frac{1}{\lambda_{0}} \sum_{j=1}^{\infty} \lambda_{0}^{-j} j\left|K_{j}\right|<1
$$

Then for any initial sequence $\phi_{j}, j \leq 0$, the solution of (1.1), (1.2) satisfies the condition

$$
\lim _{n \rightarrow \infty} \lambda_{0}^{-n} x_{n}=Q_{\lambda_{0}}(\phi)
$$

where

$$
\begin{aligned}
& Q_{\lambda_{0}}(\phi)=\frac{L_{\lambda_{0}}(\phi)}{1+\gamma_{\lambda_{0}}}, \quad \gamma_{\lambda_{0}}=\frac{1}{\lambda_{0}} \sum_{j=1}^{\infty} \lambda_{0}^{-j} j K_{j}, \\
& L_{\lambda_{0}}(\phi)=\phi_{0}+\frac{1}{\lambda_{0}} \sum_{j=1}^{\infty} \lambda_{0}^{-j} K_{j}\left(\sum_{r=-j}^{-1} \lambda_{0}^{-r} \phi_{r}\right) .
\end{aligned}
$$

The proof of Theorem 1.1 follows from [5] where, in particular, it is shown that the sequence

$$
z_{n}=\lambda_{0}^{-n} x_{n}-Q_{\lambda_{0}}(\phi)
$$

is a solution of the linear difference equation

$$
z_{n}=-\frac{1}{\lambda_{0}} \sum_{j=1}^{\infty} \lambda_{0}^{-j} K_{j}\left(\sum_{r=n-j}^{n-1} z_{r}\right), \quad n>0
$$

and by condition (1.4) $z_{n}$, defined by (1.7), converges to zero that is equivalent to (1.5).

Two following questions arise here.

Firstly, it is clear that condition (1.4) is a sufficient condition for asymptotic stability of the trivial solution of (1.8). But is condition (1.4) a unique or the best sufficient condition? 
Secondly, why only a positive root of (1.3) is considered here? Which is a situation in the case of negative or complex root?

Below it is shown that condition (1.4) of Theorem 1.1 can be weaken and the negative and complex roots of (1.3) also can be useful for investigation of asymptotic behavior of the solution of (1.1), (1.2).

\section{Improvement of the known result}

Rewrite equation (1.8) in the form

$$
z_{n}=\sum_{l=1}^{\infty} a_{l} z_{n-l}, \quad n>0, \quad a_{l}=-\frac{1}{\lambda_{0}} \sum_{j=l}^{\infty} \lambda_{0}^{-j} K_{j}
$$

Different sufficient conditions for asymptotic stability of the trivial solution of difference Volterra equation type of $(2.1)$ were obtained in $[1-4,13]$ via the general method of Lyapunov functionals construction.

In particular, if

$$
\sum_{l=1}^{\infty}\left|a_{l}\right|<1
$$

then the trivial solution of (2.1) is asymptotically stable [1]. Condition (2.2) is weaker than (1.4). Really,

$$
\sum_{l=1}^{\infty}\left|a_{l}\right| \leq \frac{1}{\lambda_{0}} \sum_{l=1}^{\infty} \sum_{j=l}^{\infty} \lambda_{0}^{-j}\left|K_{j}\right|=\frac{1}{\lambda_{0}} \sum_{j=1}^{\infty} \sum_{l=1}^{j} \lambda_{0}^{-j}\left|K_{j}\right|=\frac{1}{\lambda_{0}} \sum_{j=1}^{\infty} \lambda_{0}^{-j} j\left|K_{j}\right|<1 .
$$

Another sufficient condition for asymptotic stability of the trivial solution of difference Volterra equation (2.1) has [1] the following form: if $2 \alpha-1<\beta<1$, where

$$
\beta=\sum_{l=1}^{\infty} a_{l}, \quad \alpha=\sum_{l=1}^{\infty} B_{l}, \quad B_{l}=\left|\sum_{j=l+1}^{\infty} a_{j}\right|,
$$

then the trivial solution of (2.1) is asymptotically stable.

So, the following theorem holds.

Theorem 2.1. Let $\lambda_{0}$ be a positive root of characteristic equation (1.3) that satisfies the property

$$
\frac{1}{\lambda_{0}} \sum_{l=1}^{\infty}\left|\sum_{j=l}^{\infty} \lambda_{0}^{-j} K_{j}\right|<1
$$


4 A new view on one problem

or the property

$$
\begin{gathered}
2 \alpha-1<\beta<1, \\
\alpha=\frac{1}{\lambda_{0}} \sum_{l=1}^{\infty}\left|\sum_{j=l+1}^{\infty}(j-l) \lambda_{0}^{-j} K_{j}\right|, \quad \beta=-\frac{1}{\lambda_{0}} \sum_{l=1}^{\infty} \sum_{j=l}^{\infty} \lambda_{0}^{-j} K_{j} .
\end{gathered}
$$

Then for any initial sequence $\phi_{j}, j \leq 0$, the solution of (1.1), (1.2) satisfies condition (1.5), (1.6).

From (2.3) it follows that condition (2.5) is weaker than (1.4). To compare conditions (1.4), (2.5), and (2.6) consider the following example.

Example 2.2. Consider the difference equation

$$
\Delta x_{n}=a x_{n}+K_{1} x_{n-1}+K_{2} x_{n-2}
$$

Auxiliary difference equation (2.1) in this case has the form

$$
z_{n}=-\left(K_{1} \lambda_{0}^{-2}+K_{2} \lambda_{0}^{-3}\right) z_{n-1}-K_{2} \lambda_{0}^{-3} z_{n-2}
$$

Conditions (1.4), (2.5), and (2.6) are correspondingly

$$
\begin{gathered}
\left|K_{1}\right| \lambda_{0}^{-2}+2\left|K_{2}\right| \lambda_{0}^{-3}<1, \\
\left|K_{1} \lambda_{0}^{-2}+K_{2} \lambda_{0}^{-3}\right|+\left|K_{2}\right| \lambda_{0}^{-3}<1, \\
-1<K_{1} \lambda_{0}^{-2}+2 K_{2} \lambda_{0}^{-3}<1-2\left|K_{2}\right| \lambda_{0}^{-3} .
\end{gathered}
$$

It is well known also [13] that the necessary and sufficient condition for asymptotic stability of the trivial solution of (2.8) is

$$
\left|K_{1} \lambda_{0}^{-2}+K_{2} \lambda_{0}^{-3}\right|<1+K_{2} \lambda_{0}^{-3}, \quad\left|K_{2}\right| \lambda_{0}^{-3}<1 .
$$

One can see that condition (2.12) follows from each of conditions (2.9), (2.10), and (2.11). From each of these conditions it follows also that $1+\gamma_{\lambda_{0}}=1+K_{1} \lambda_{0}^{-2}+2 K_{2} \lambda_{0}^{-3}>0$, so $Q_{\lambda_{0}}(\phi)$ in (1.6) is defined. 


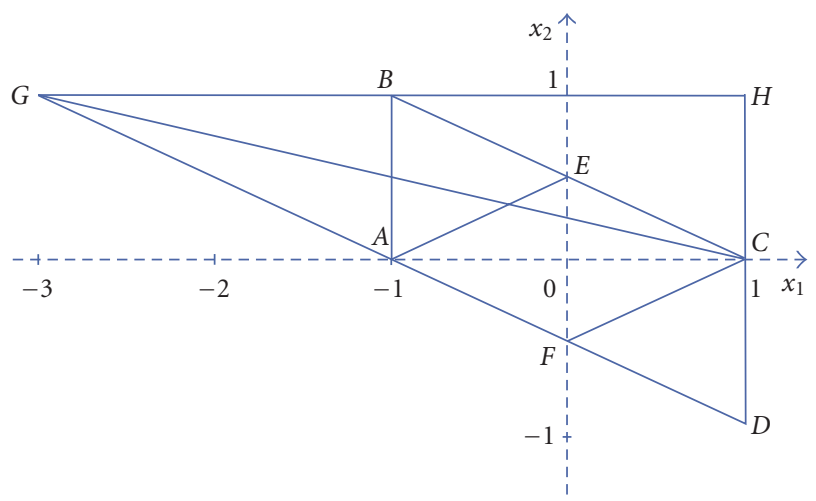

Figure 2.1. Different stability regions.

On Figure 2.1 stability regions for (2.8) are shown constructed by conditions (2.9) (region AECF), (2.10) (region ABCD), (2.11) (region GCD), and (2.12) (region GHD) in the space $\left(x_{1}, x_{2}\right)$, where $x_{1}=K_{1} \lambda_{0}^{-2}, x_{2}=K_{2} \lambda_{0}^{-3}$.

\section{Different situations with roots of the characteristic equation}

To demonstrate the different situations of the use not only positive but also negative and complex roots of characteristic equation (1.3) consider the simple difference equation

$$
\begin{gathered}
\Delta x_{n}=a x_{n}+b x_{n-1}, \quad n=0,1, \ldots, \\
x_{j}=\phi_{j}, \quad j=-1,0 .
\end{gathered}
$$

The corresponding characteristic equation is

$$
\lambda-1=a+b \lambda^{-1}
$$

The following theorem deals with behavior of the sequences $x_{n}$ and $y_{n}=\lambda_{0}^{-n} x_{n}$, where $x_{n}$ is a solution of (3.1) and $\lambda_{0}$ is a root of characteristic equation (3.2).

THEOREM 3.1. There are four different situations with a solution of (3.1).

(1) If

$$
a+1 \neq 0, \quad(a+1)^{2}+4 b>0,
$$

then

$$
\lim _{n \rightarrow \infty} y_{n}=Q_{\lambda_{0}}(\phi)
$$


6 A new view on one problem

where

$$
\begin{gathered}
Q_{\lambda_{0}}(\phi)=\frac{L_{\lambda_{0}}(\phi)}{1+\lambda_{0}^{-2} b}, \quad L_{\lambda_{0}}(\phi)=\phi_{0}+\lambda_{0}^{-1} b \phi_{-1}, \\
\lambda_{0}=\frac{a+1}{2}\left(1+\sqrt{1+\frac{4 b}{(a+1)^{2}}}\right) .
\end{gathered}
$$

(2) If

$$
a+1=0, \quad b>0,
$$

then $\lambda_{0}= \pm \sqrt{b}$ and

$$
y_{2 k}=\phi_{0}, \quad y_{2 k+1}=\lambda_{0} \phi_{-1}, \quad k=0,1, \ldots
$$

(3) If

$$
a+1 \neq 0, \quad(a+1)^{2}+4 b=0,
$$

then

$$
y_{n}=\phi_{0}+n L_{\lambda_{0}}(\phi), \quad n=0,1, \ldots,
$$

where $L_{\lambda_{0}}(\phi)$ is defined by (3.5) and $\lambda_{0}=(1 / 2)(a+1)$.

(4) If

$$
(a+1)^{2}+4 b<0
$$

then

$$
\left|y_{n}-Q_{\lambda_{0}}(\phi)\right|=\left|\phi_{0}-Q_{\lambda_{0}}(\phi)\right|, \quad n=0,1, \ldots
$$

where

$$
Q_{\lambda_{0}}(\phi)=\frac{\phi_{0}}{2} \mp i \frac{\phi_{0}(a+1)+2 b \phi_{-1}}{2 \sqrt{\left|(a+1)^{2}+4 b\right|}},
$$

and $\lambda_{0}$ is one of two conjugate complex roots

$$
\lambda_{0}=\frac{a+1 \pm i \sqrt{\left|(a+1)^{2}+4 b\right|}}{2}, \quad i=\sqrt{-1},
$$

of characteristic equation (3.2). It means that the values of the process $y_{n}$ are located in a complex plane on the circle with the center $Q_{\lambda_{0}}(\phi)$ and the radius $r=\left|\phi_{0}-Q_{\lambda_{0}}(\phi)\right|$. This circle includes the points 0 and $\phi_{0}$. 
Proof. (1) Let us suppose that condition (3.3) holds. Put

$$
z_{n}=y_{n}-Q_{\lambda_{0}}(\phi), \quad y_{n}=\lambda_{0}^{-n} x_{n},
$$

where $x_{n}$ is a solution of (3.1) and $\lambda_{0}$ is a root of characteristic equation (3.2).

By condition (3.3), (3.2) has two real roots

$$
\lambda_{1,2}=\frac{a+1 \pm \sqrt{(a+1)^{2}+4 b}}{2}=\frac{a+1}{2}\left(1 \pm \sqrt{1+\frac{4 b}{(a+1)^{2}}}\right) .
$$

From (2.1) it follows that sequence (3.15) satisfies the equation

$$
z_{n}=-\lambda_{0}^{-2} b z_{n-1}, \quad n=0,1, \ldots
$$

The necessary and sufficient condition for asymptotic stability of the trivial solution of (3.17) is

$$
\left|\lambda_{0}^{-2} b\right|<1
$$

From (3.2) it follows that condition (3.18) is equivalent to $\left|1-(a+1) \lambda_{0}^{-1}\right|<1$ or

$$
\lambda_{0}(a+1)^{-1}>\frac{1}{2}
$$

It is easy to see that from two roots (3.16) of (3.2) root (3.6) only satisfies condition (3.19). So (3.4) is proven.

(2) By conditions (3.7) from (3.2) it follows that $\lambda_{0}^{-2} b=1$. Equation (3.17) takes the form $z_{n}=-z_{n-1}$. Therefore, $z_{n}=(-1)^{n} z_{0}, n=1,2, \ldots$ Via (3.15), (3.5) from here we have

$$
\begin{aligned}
y_{n} & =Q_{\lambda_{0}}(\phi)+(-1)^{n}\left[\phi_{0}-Q_{\lambda_{0}}(\phi)\right] \\
& =(-1)^{n} \phi_{0}+\frac{1}{2}\left[1-(-1)^{n}\right]\left[\phi_{0}+\left(\lambda_{0}^{-2} b\right) \lambda_{0} \phi_{-1}\right] \\
& =\frac{1}{2}\left[1+(-1)^{n}\right] \phi_{0}+\frac{\lambda_{0}}{2}\left[1-(-1)^{n}\right] \phi_{-1}
\end{aligned}
$$

that is equivalent to (3.8).

(3) By condition (3.9) the solution of (3.2) is $\lambda_{0}=(1 / 2)(a+1)$. From here and (3.9) it follows that $1+\lambda_{0}^{-2} b=0$ and, therefore, $Q_{\lambda_{0}}(\phi)$ in (3.5) is undefined. It means that sequence (3.15) undefined too. Using $y_{j}=\lambda_{0}^{-j} x_{j}, j=0,1, \ldots$, (3.1), (3.2), and $\lambda_{0}^{-1} b=-\lambda_{0}$, we have

$$
\begin{aligned}
\Delta x_{j}-a x_{j}-b x_{j-1} & =\Delta\left(\lambda_{0}^{j} y_{j}\right)-a \lambda_{0}^{j} y_{j}-b \lambda_{0}^{j-1} y_{j-1} \\
& =\lambda_{0}^{j}\left[\lambda_{0} \Delta y_{j}+\left(\lambda_{0}-1-a\right) y_{j}-b \lambda_{0}^{-1} y_{j-1}\right] \\
& =\lambda_{0}^{j+1}\left[\Delta y_{j}-\Delta y_{j-1}\right]=0 .
\end{aligned}
$$




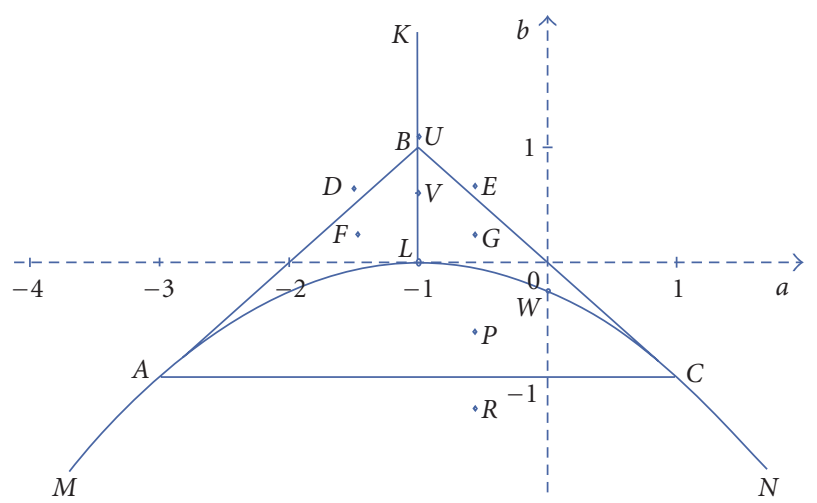

Figure 3.1. Regions with different behavior of $x_{n}$ and $y_{n}$.

From here via (3.5) it follows that

$$
\Delta y_{j}=\Delta y_{j-1}=y_{0}-y_{-1}=x_{0}-\lambda_{0} x_{-1}=\phi_{0}+\lambda_{0}^{-1} b \phi_{-1}=L_{\lambda_{0}}(\phi)
$$

or $y_{j}=y_{j-1}+L_{\lambda_{0}}(\phi)$. Summing this equality with respect to $j=1,2, \ldots, n$, we obtain (3.10).

(4) Let us suppose now that condition (3.11) holds. Then the conjugate complex roots of (3.2) are defined by (3.14) and satisfy the condition $\left|\lambda_{0}\right|^{2}=-b=|b|$ or $\left|\lambda_{0}^{-2} b\right|=1$. From (3.17) it follows that process (3.15) satisfies the equation $\left|z_{n}\right|=\left|z_{n-1}\right|$ or $\left|z_{n}\right|=\left|z_{0}\right|$. It is equivalent to (3.12). Now it is enough to show that $Q_{\lambda_{0}}(\phi)$ defined by (3.5) equals $Q_{\lambda_{0}}(\phi)$ defined by (3.13). Really, putting $\delta=\sqrt{\left|(a+1)^{2}+4 b\right|}$, from (3.14) we obtain $2 \lambda_{0}-$ $(a+1)= \pm i \delta$. Using (3.2), (3.5), and (3.14), one can transform $Q_{\lambda_{0}}(\phi)$ by the following way:

$$
\begin{aligned}
Q_{\lambda_{0}}(\phi) & =\frac{L_{\lambda_{0}}(\phi)}{2-\lambda_{0}^{-1}(a+1)}=\frac{\lambda_{0} L_{\lambda_{0}}(\phi)}{2 \lambda_{0}-(a+1)}=\frac{\lambda_{0} L_{\lambda_{0}}(\phi)}{ \pm i \delta}=\frac{2 i \lambda_{0} L_{\lambda_{0}}(\phi)}{\mp 2 \delta} \\
& =\frac{i\left(\phi_{0}(a+1) \pm i \phi_{0} \delta+2 b \phi_{-1}\right)}{\mp 2 \delta}=\frac{\phi_{0}}{2} \mp i \frac{\phi_{0}(a+1)+2 b \phi_{-1}}{2 \delta} .
\end{aligned}
$$

The theorem is proven.

Four regions described in Theorem 3.1 are shown on Figure 3.1: (1) at the left of the curve $K L M$ and from the right of the curve $K L N$; (2) the line $K L$; (3) the curve $M L N$; (4) under the curve $M L N$. The point $L$ with the coordinates $a=-1, b=0$ is excluded from the consideration since in this point $\lambda_{0}=0$. The inside of the triangle $A B C$ is the region of asymptotic stability of the trivial solution of (3.1).

Below on Figures 3.2, 3.4, and 3.5 the first situation from Theorem 3.1 is shown.

On Figure 3.2 the trajectories of the processes $x_{n}$ and $y_{n}$ are shown in the point $D$ (it is shown on Figure 3.1) with the coordinates $a=-1.5, b=0.65$. Here $\phi_{-1}=2, \phi_{0}=0.5$, $\lambda_{0}=-1.094$ (a negative root). The point $P$ does not belong to the stability region (the 


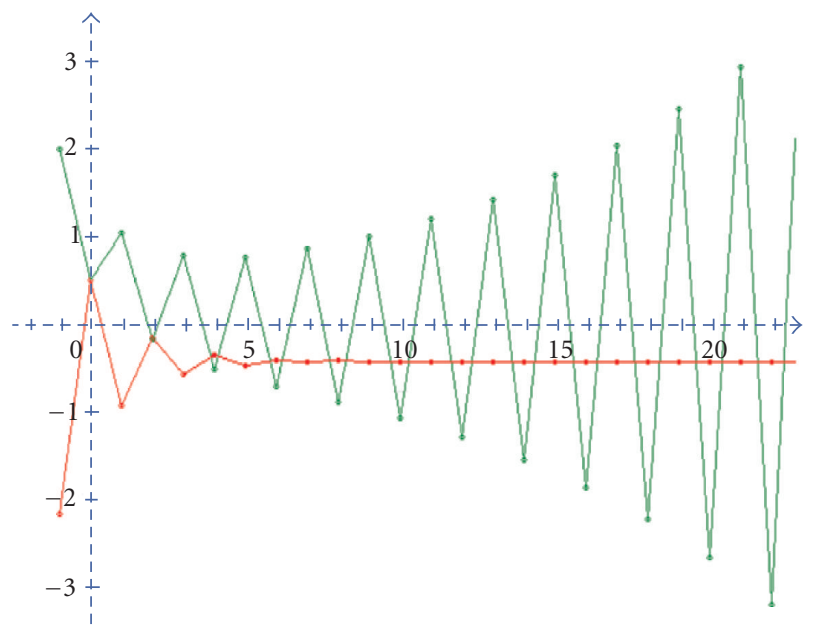

Figure 3.2. Regions with different behavior of $x_{n}$ and $y_{n}$ in the point $D$.

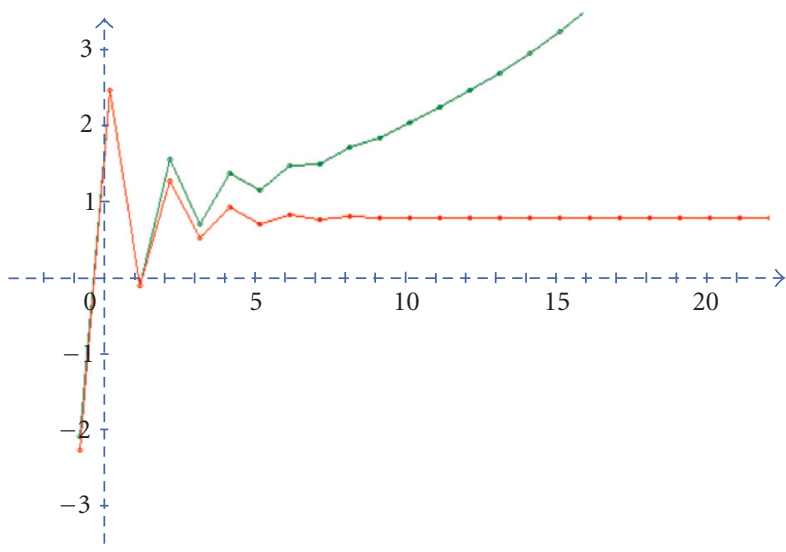

Figure 3.3. Behavior of $x_{n}$ and $y_{n}$ in the point $E$.

triangle $A B C$ ) of the trivial solution of (3.1), so the process $x_{n}$ (green) goes to $\pm \infty$. The process $y_{n}$ (red) enough quickly converges to $Q_{\lambda_{0}}(\phi)=-0.446$.

On Figure 3.3 the similar trajectories of the processes $x_{n}$ and $y_{n}$ are shown in the point $E$ (Figure 3.1). Here $a=-0.5, b=0.65, \phi_{-1}=-2, \phi_{0}=2.5, \lambda_{0}=1.094$ (a positive root), the process $x_{n}$ (green) goes to $+\infty$, the process $y_{n}$ (red) quickly converges to $Q_{\lambda_{0}}(\phi)=$ 0.850 .

On Figure 3.4 the trajectories of the processes $x_{n}$ and $y_{n}$ are shown in the point $F$ (Figure 3.1) with the coordinates $a=-1.5, b=0.25$. Here $\phi_{-1}=3, \phi_{0}=-1.5, \lambda_{0}=$ -0.809 (a negative root). The point $F$ belongs to the stability region (the triangle $A B C$ ) 


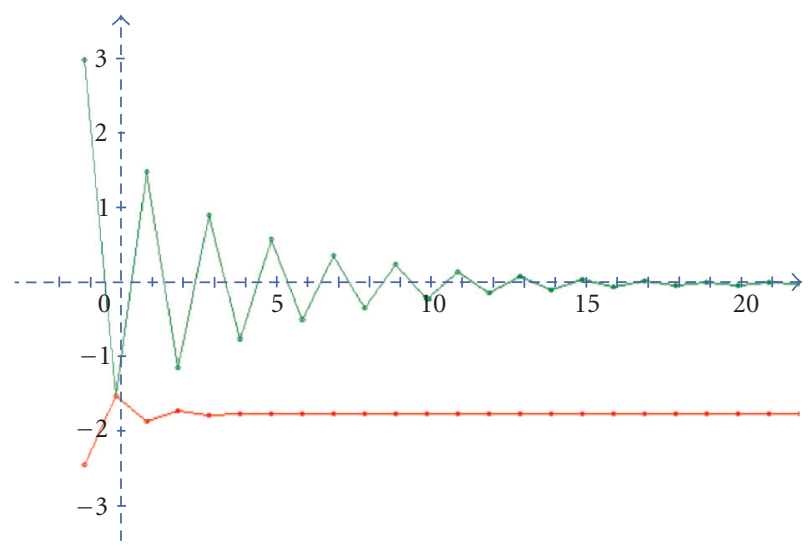

Figure 3.4. Behavior of $x_{n}$ and $y_{n}$ in the point $F$.

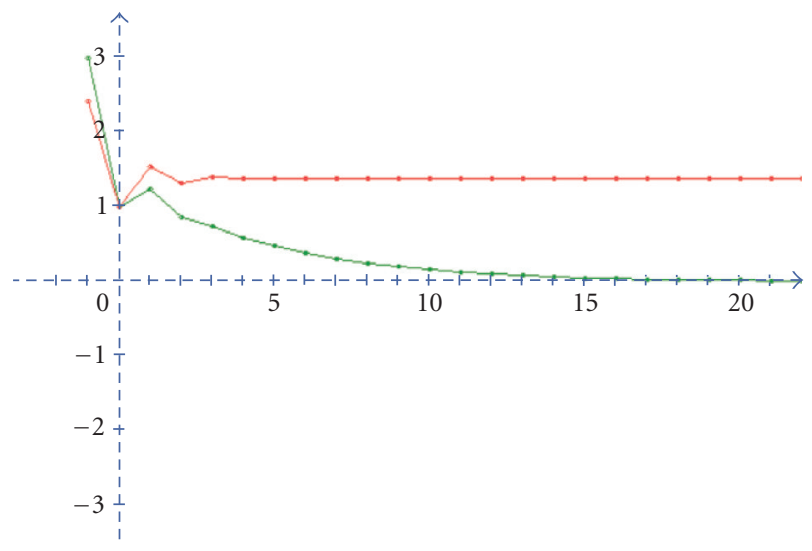

Figure 3.5. Behavior of $x_{n}$ and $y_{n}$ in the point $G$.

of the trivial solution of (3.1), so, the process $x_{n}$ (green) converges to zero. The process $y_{n}$ (red) quickly converges to $Q_{\lambda_{0}}(\phi)=-1.756$.

On Figure 3.5 the similar trajectories of the processes $x_{n}$ and $y_{n}$ are shown in the point $G$ (Figure 3.1). Here $a=-0.5, b=0.25, \phi_{-1}=3, \phi_{0}=1, \lambda_{0}=0.809$ (a positive root), the process $x_{n}$ (green) converges to zero, the process $y_{n}$ (red) quickly converges to $Q_{\lambda_{0}}(\phi)=$ 1.394.

On Figures 3.6 and 3.7 the second situation from Theorem 3.1 is shown.

On Figure 3.6 the trajectories of the processes $x_{n}$ and $y_{n}$ are shown in the point $U$ (Figure 3.1) with the coordinates $a=-1, b=1.1$. Here $\phi_{-1}=1.5, \phi_{0}=-1, \lambda_{0}=-1.049$ (a negative root). The point $U$ does not belong to the stability region (the triangle $A B C$ ) of the trivial solution of (3.1), so the process $x_{n}$ (green) goes to $\pm \infty$. The process $y_{n}$ (red) has two values: $\phi_{0}=-1$ and $\lambda_{0} \phi_{-1}=-1.573$. 


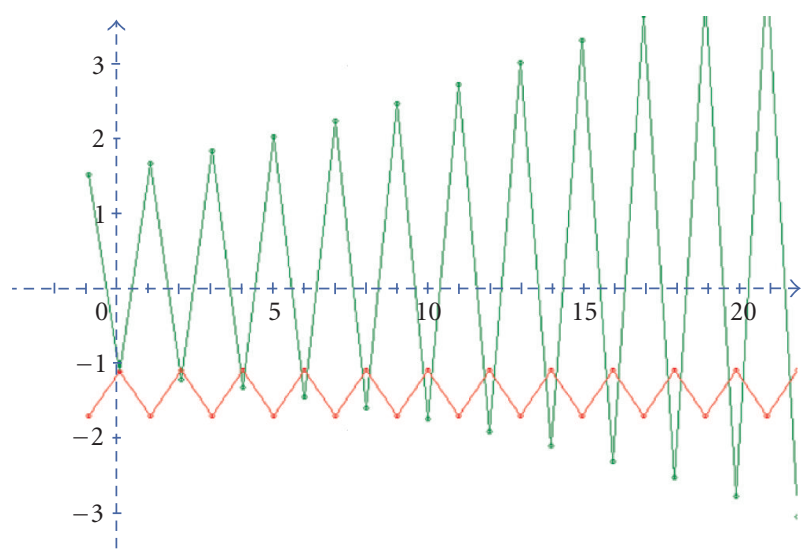

Figure 3.6. Behavior of $x_{n}$ and $y_{n}$ in the point $U$.

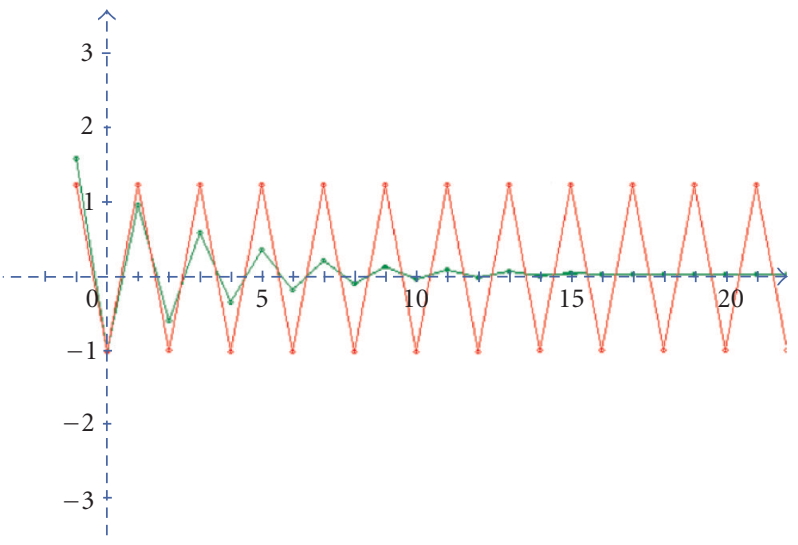

Figure 3.7. Behavior of $x_{n}$ and $y_{n}$ in the point $V$.

On Figure 3.7 the similar trajectories of the processes $x_{n}$ and $y_{n}$ are shown in the point $V$ (Figure 3.1). Here $a=-1, b=0.6, \phi_{-1}=1.5, \phi_{0}=-1, \lambda_{0}=0.775$ (a positive root), the process $x_{n}$ (green) converges to zero, the process $y_{n}$ (red) has two values: $\phi_{0}=-1$ and $\lambda_{0} \phi_{-1}=1.162$.

On Figures 3.8 and 3.9 the third situation from Theorem 3.1 is shown.

On Figure 3.8 the trajectories of the processes $x_{n}$ and $y_{n}$ are shown in the point $W$ (Figure 3.1) with the coordinates $a=0, b=-0.25$. Here $\phi_{-1}=3.5, \phi_{0}=1.6, \lambda_{0}=0.5$ (a positive root), $L_{\lambda_{0}}(\phi)=-0.15$. The point $W$ belongs to the stability region (the triangle $A B C$ ) of the trivial solution of (3.1), so the process $x_{n}$ (green) converges to zero. The process $y_{n}$ (red) is a straight line.

On Figure 3.9 the trajectories of the processes $x_{n}$ and $y_{n}$ are shown in the point $A$ (Figure 3.1) with the coordinates $a=-3, b=-1$. Here $\phi_{-1}=1.2, \phi_{0}=-1, \lambda_{0}=-1$ 


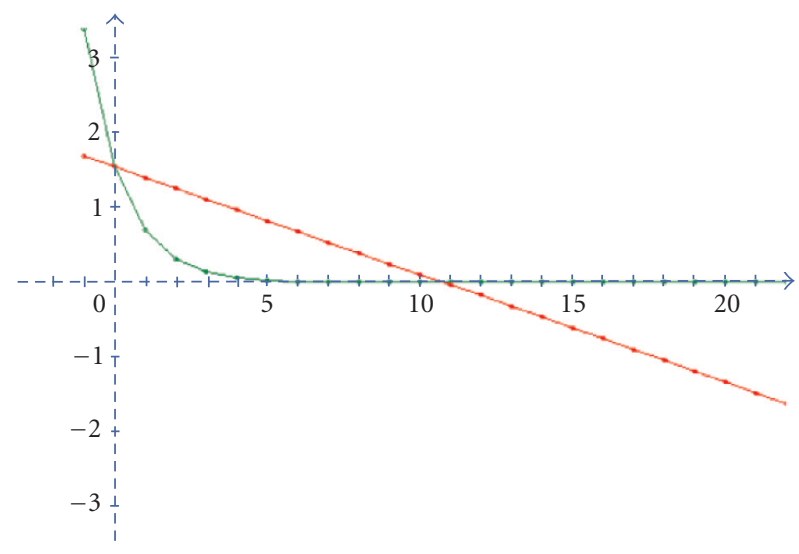

Figure 3.8. Behavior of $x_{n}$ and $y_{n}$ in the point $W$.

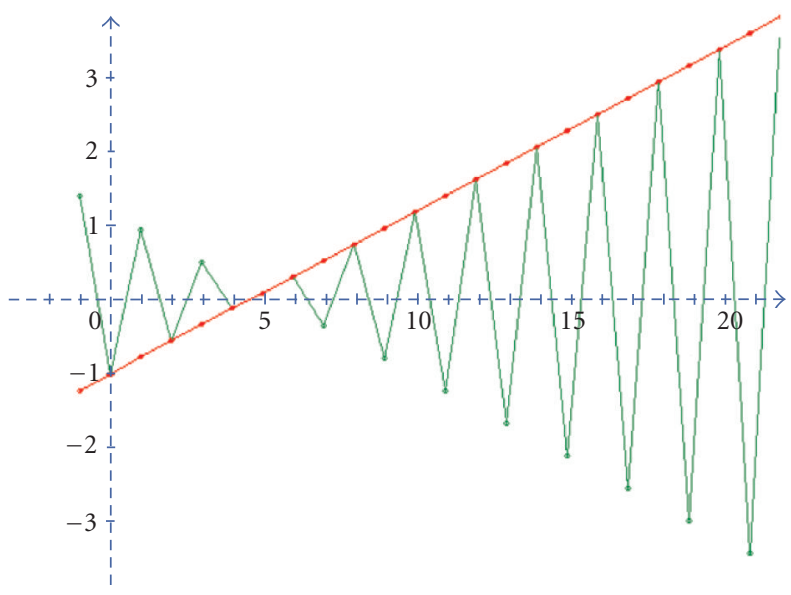

Figure 3.9. Behavior of $x_{n}$ and $y_{n}$ in the point $A$.

(a negative root), $L_{\lambda_{0}}(\phi)=0.2$. The point $A$ does not belong to the stability region (the triangle $A B C$ ) of the trivial solution of (3.1), so the process $x_{n}$ (green) goes to $\pm \infty$. The process $y_{n}$ (red) is a straight line.

On Figures 3.10 and 3.11 the fourth situation from Theorem 3.1 is shown.

On Figure 3.10(a) the trajectory of the complex process $y_{n}$ is shown in the point $P$ (Figure 3.1) with the coordinates $a=-0.5, b=-0.6$. Here $\phi_{-1}=-3, \phi_{0}=3$. One can see that the values of the process $y_{n}$ are located in the complex plane on the circle with radius $r=2.297$ and the center $Q_{\lambda_{0}}(\phi)=1.5-i 1.739$ (green) if $\lambda_{0}=0.25+i 0.733$ and $Q_{\lambda_{0}}(\phi)=1.5+i 1.739$ (red) if $\lambda_{0}=0.25-i 0.733$. On Figure $3.10(\mathrm{~b})$ the trajectory of the process $x_{n}$ is shown in the same point $P$ (Figure 3.1). This point belongs to the stability 


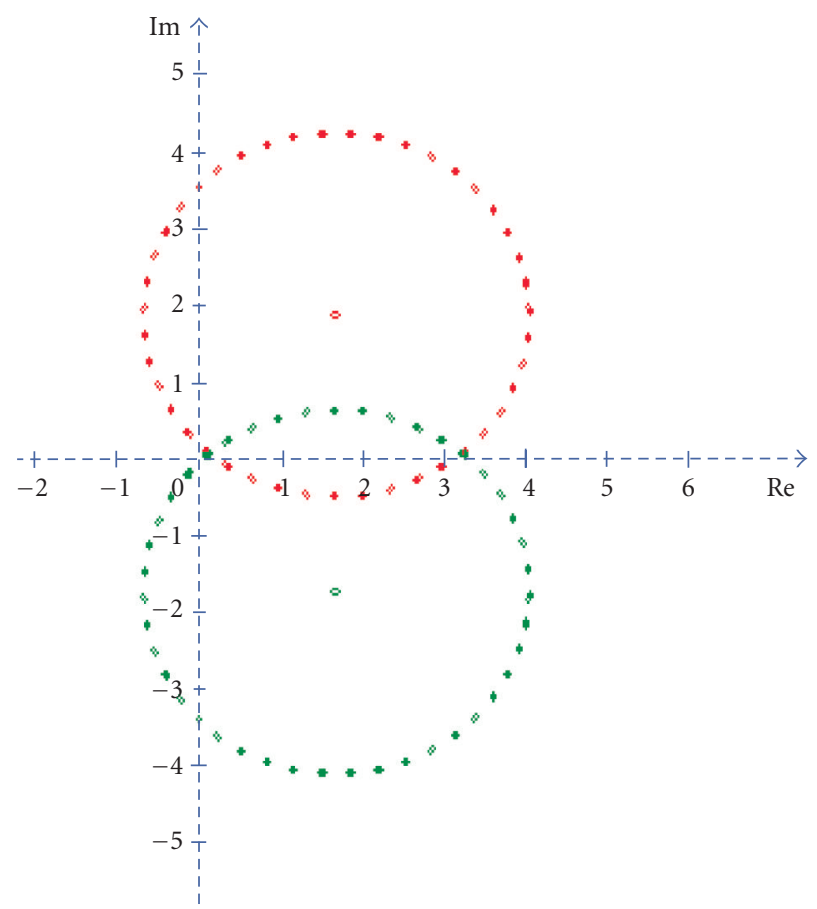

(a)

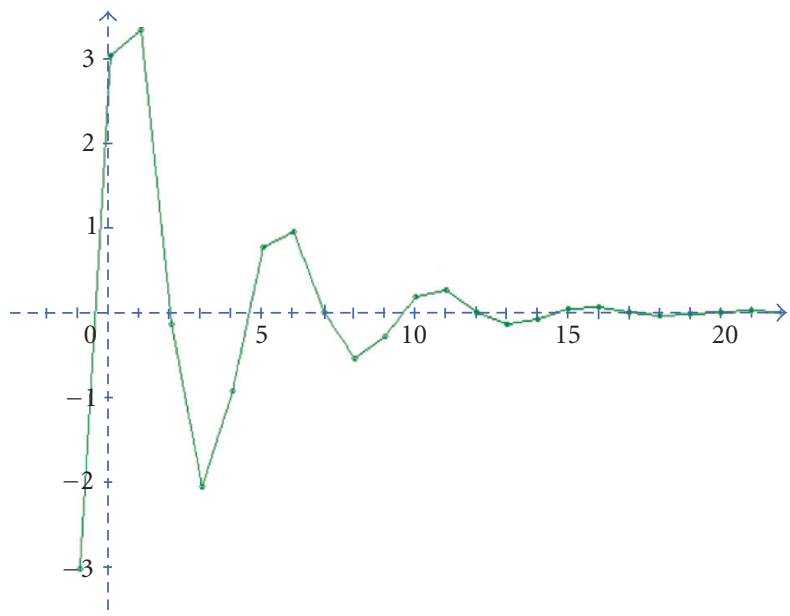

(b)

Figure 3.10. (a) Behavior of $y_{n}$ in the point $P$; (b) behavior of $x_{n}$ in the point $P$. 
14 A new view on one problem

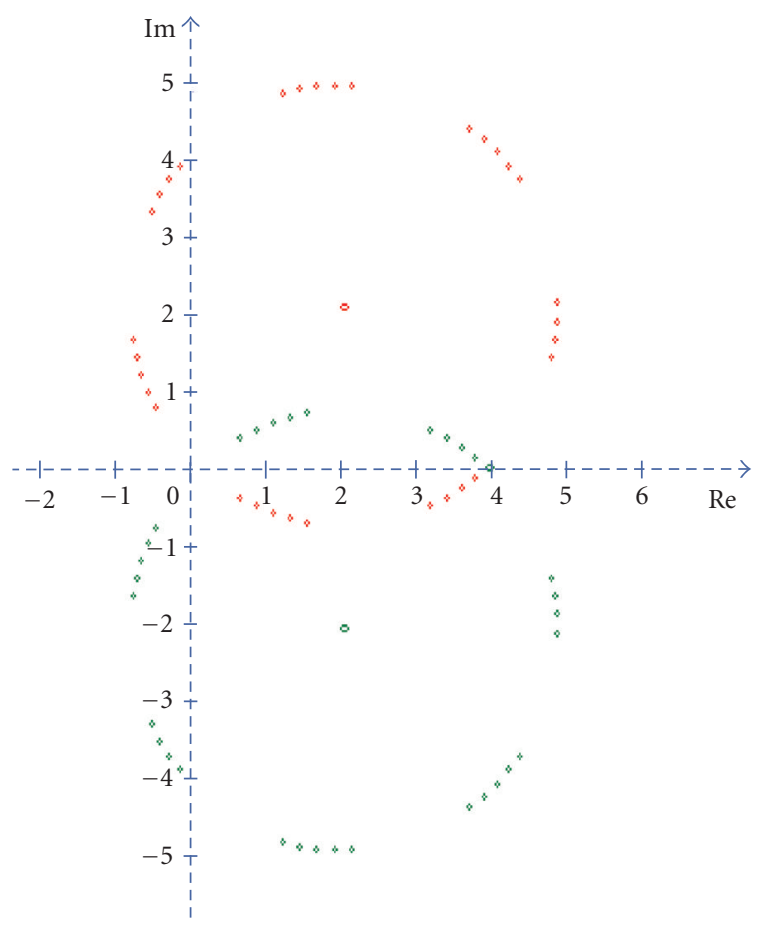

(a)

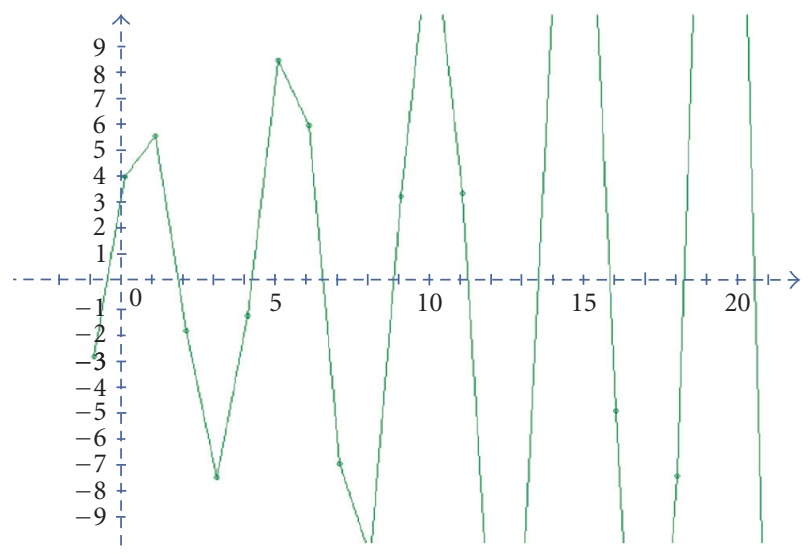

(b)

Figure 3.11. (a) Behavior of $y_{n}$ in the point $R$; (a) behavior of $x_{n}$ in the point $R$. 
region (the triangle $A B C$ ) of the trivial solution of (3.1), so the process $x_{n}$ converges to zero.

On Figure 3.11(a) the trajectory of the complex process $y_{n}$ is shown in the point $R$ (Figure 3.1) with the coordinates $a=-0.5, b=-1.2$. Here $\phi_{-1}=-3, \phi_{0}=4$. One can see that the values of the process $y_{n}$ are located in the complex plane on the circle with radius $r=2.941$ and the center $Q_{\lambda_{0}}(\phi)=2-i 2.157$ (green) if $\lambda_{0}=0.25+i 1.067$ and $Q_{\lambda_{0}}(\phi)=$ $2+i 2.157$ (red) if $\lambda_{0}=0.25-i 1.067$. On Figure 3.11 (b) the trajectory of the process $x_{n}$ is shown in the same point $R$ (Figure 3.1). This point does not belong to the stability region (the triangle $A B C$ ) of the trivial solution of (3.1), so the process $x_{n}$ goes to $\pm \infty$.

\section{Conclusion}

In this paper it is shown that the known results type of Theorem 1.1 (see [5-12]) can be improved similar to Theorem 2.1 by virtue of different stability conditions obtained via general method of Lyapunov functionals construction $[1-4,13]$. On the other hand, it is noted that the results of the papers [5-12] in general case can be essentially extended similar to Theorem 3.1 via consideration of not only positive but also negative and complex roots of the corresponding characteristic equation. These ideas can be applied both for difference and for functional-differential equations.

\section{References}

[1] V. Kolmanovskii and L. Shaikhet, General method of Lyapunov functionals construction for stability investigation of stochastic difference equations, Dynamical Systems and Applications, World Sci. Ser. Appl. Anal., vol. 4, World Scientific, New Jersey, 1995, pp. 397-439.

[2] - Some peculiarities of the general method of Lyapunov functionals construction, Applied Mathematics Letters 15 (2002), no. 3, 355-360.

[3] _ About one application of the general method of Lyapunov functionals construction, International Journal of Robust and Nonlinear Control 13 (2003), no. 9, 805-818, special issue on time-delay systems.

[4] - About some features of general method of Lyapunov functionals construction, Stability and Control: Theory and Applications 6 (2004), no. 1, 49-76.

[5] I.-G. E. Kordonis and Ch. G. Philos, On the behavior of the solutions for linear autonomous neutral delay difference equations, Journal of Difference Equations and Applications 5 (1999), no. 3, 219233.

[6] The behavior of solutions of linear integro-differential equations with unbounded delay, Computers \& Mathematics with Applications 38 (1999), no. 2, 45-50.

[7] I.-G. E. Kordonis, Ch. G. Philos, and I. K. Purnaras, Some results on the behavior of the solutions of a linear delay difference equation with periodic coefficients, Applicable Analysis 69 (1998), no. 1-2, 83-104.

[8] _ On the behavior of solutions of linear neutral integrodifferential equations with unbounded delay, Georgian Mathematical Journal 11 (2004), no. 2, 337-348.

[9] Ch. G. Philos, Asymptotic behaviour, nonoscillation and stability in periodic first-order linear delay differential equations, Proceedings of the Royal Society of Edinburgh. Section A 128 (1998), no. $6,1371-1387$.

[10] Ch. G. Philos and I. K. Purnaras, Periodic first order linear neutral delay differential equations, Applied Mathematics and Computation 117 (2001), no. 2-3, 203-222. 


\section{A new view on one problem}

[11] _ An asymptotic result for some delay difference equations with continuous variable, Advances in Difference Equations 2004 (2004), no. 1, 1-10.

[12] _ Asymptotic properties, nonoscillation, and stability for scalar first order linear autonomous neutral delay differential equations, Electronic Journal of Differential Equations 2004 (2004), no. 3, 1-17.

[13] L. Shaikhet, Necessary and sufficient conditions of asymptotic mean square stability for stochastic linear difference equations, Applied Mathematics Letters 10 (1997), no. 3, 111-115.

L. Shaikhet: Department of Higher Mathematics, Donetsk State University of Management, Chelyuskintsev street 163-A, Donetsk 83015, Ukraine

E-mail addresses: leonid@dsum.edu.ua; leonid.shaikhet@usa.net 


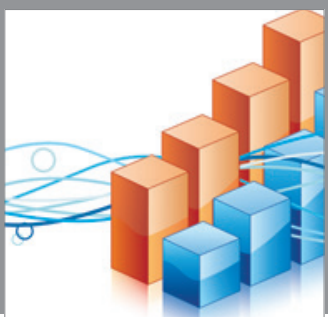

Advances in

Operations Research

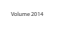

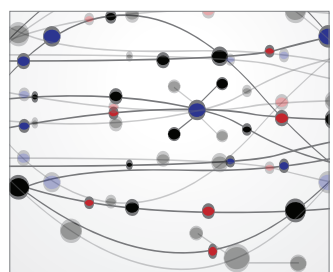

\section{The Scientific} World Journal
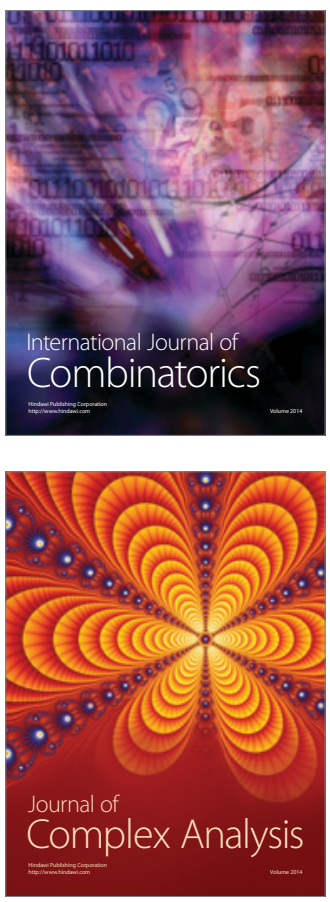

International Journal of

Mathematics and

Mathematical

Sciences
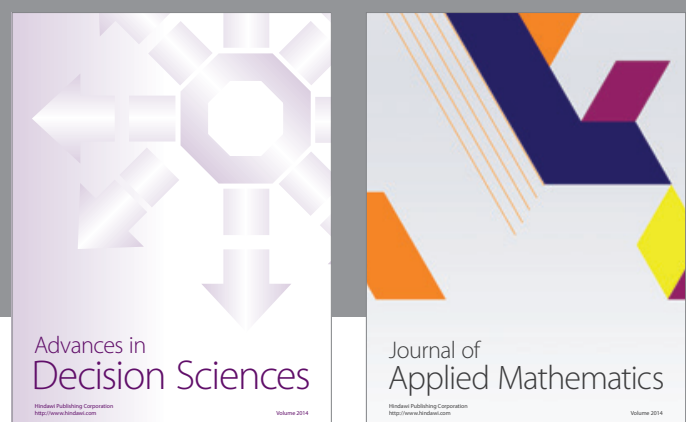

Journal of

Applied Mathematics
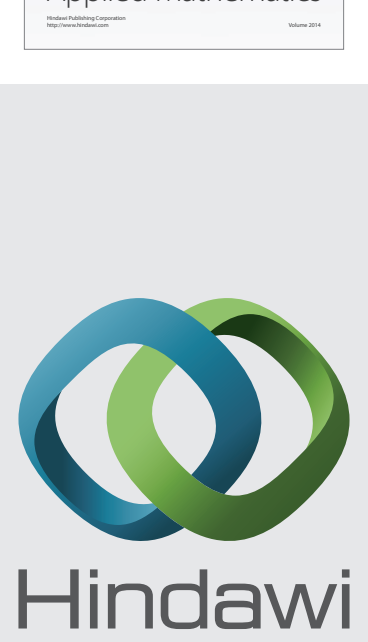

Submit your manuscripts at http://www.hindawi.com
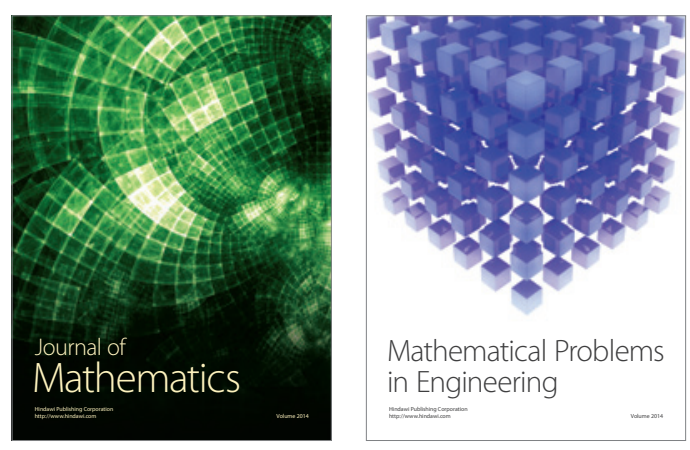

Mathematical Problems in Engineering
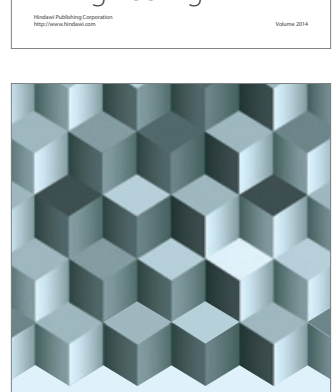

Journal of

Function Spaces
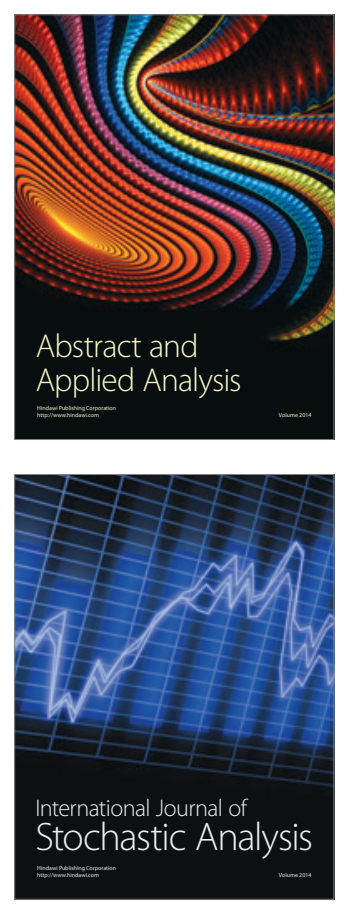

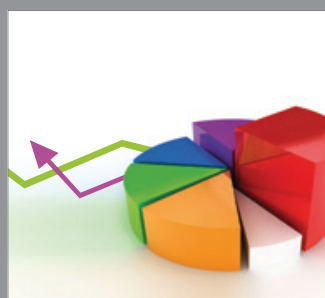

ournal of

Probability and Statistics

Promensencen
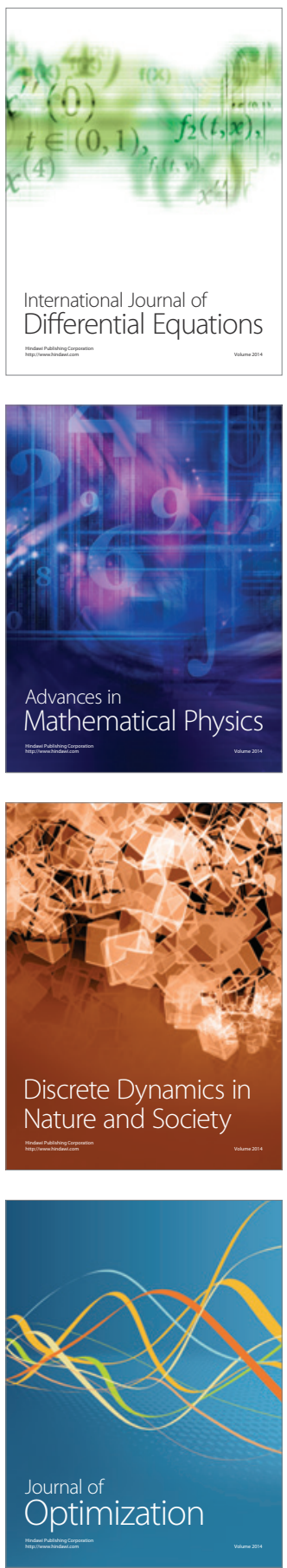